\title{
Eosinophilic Esophagitis, Eosinophilic Gastroenteritis, and Eosinophilic Colitis: Common Mechanisms and Differences between East and West
}

\author{
Shunji Ishihara ${ }^{a}$ Yoshikazu Kinoshita $^{a}$ Alain Schoepfer ${ }^{b}$ \\ ${ }^{a}$ Department of Internal Medicine II, Shimane University Faculty of Medicine, Izumo, Japan; ${ }^{b}$ Division of \\ Gastroenterology and Hepatology, Centre Hospitalier Universitaire Vaudois/CHUV, Lausanne, Switzerland
}

\section{Key Words}

Eosinophilic esophagitis · Eosinophilic gastroenteritis .

Eosinophilic colitis · Eosinophils · Inflammation

\begin{abstract}
Background: Eosinophilic esophagitis (EoE), eosinophilic gastroenteritis (EGE), and eosinophilic colitis (EoC) have been diagnosed with an increasing frequency over the last decades. All these diseases share pathogenic similarities with respect to triggering by food antigens in the majority of patients once secondary causes of tissue eosinophilia have been excluded. While diagnostic criteria for EoE have been published, the exact criteria for EGE and EoC still need to be defined. This review highlights similarities and differences between affected patients when comparing the Western with the Eastern world. Summary: The incidence and prevalence of EoE are on the rise in countries from the Western and Eastern world. Very limited epidemiologic data exist regarding EGE and EoC. Characteristics of patients with eosinophilic gastrointestinal disorders regarding gender distribution, age at diagnosis, and associated comorbidities are similar when comparing Eastern with Western countries. Similar modalities are applied to diagnose EoE, EGE, and EoC
\end{abstract}

when comparing Eastern with Western countries. Additionally, comparable therapeutic measures are applied to treat eosinophilic gastrointestinal diseases in Eastern and Western countries. While EoE treatment recommendations are based on increasingly solid evidence, recommendations for the treatment of EGE and EoC are based on low evidence. Key Messages: Eosinophilic gastrointestinal diseases are diagnosed and treated using similar approaches in Eastern and Western countries. Further efforts should be undertaken to help clarify the underlying pathogenic mechanisms and to establish evidence-based diagnostic and therapeutic protocols.

(c) 2016 S. Karger AG, Basel

\section{Introduction}

Eosinophilic gastrointestinal disorders (EGIDs) are chronic inflammatory conditions characterized by dense eosinophilic inflammation of the gastrointestinal tract. They are classified according to the affected region of the gastrointestinal tract and include eosinophilic esophagitis (EoE), eosinophilic gastroenteritis (EGE), and eosinophilic colitis (EoC). The etiologic mechanisms remain to

\section{KARGER}

E-Mail karger@karger.com

www.karger.com/iid
(C) 2016 S. Karger AG, Basel

2296-9403/16/0012-0063\$39.50/0
Alain Schoepfer, MD

Centre Hospitalier Universitaire Vaudois/CHUV

Rue de Bugnon 44

CH-1011 Lausanne (Switzerland)

E-Mail Alain.schoepfer@chuv.ch 
be clarified, but recent evidence points towards foodbased antigens as a central trigger in activating the inflammatory cascades that underlie the disease pathology. Of these entities, EoE is best characterized, with eosinophilic infiltration of the esophagus being its distinguishing feature [1]. EGE is a rare disease occurring in both children and adults [2]. It is characterized by eosinophilic infiltration of the stomach or intestinal wall, leading to a variety of gastrointestinal symptoms [2]. EoC is considered to be a non-IgE-mediated allergic response to food proteins and usually affects children, although a few adult cases have also been reported. The main feature of this rare disease is extensive eosinophilic infiltration of the colon [3]. This article provides an overview of the epidemiology, diagnosis, treatment, and management of these disorders from the Japanese and Swiss perspectives.

\section{Epidemiology}

In general, EoE has been reported more frequently in Western countries than in Asia [4]. Four recent epidemiological studies focusing on the incidence of EoE have been conducted in Japan, which included patients undergoing endoscopy either due to upper gastrointestinal symptoms or as part of an annual medical check-up [58]. Altogether, the results from these studies indicate that the incidence of EoE ranges from 0.01 to $0.13 \%$. In contrast, another Japanese study reported a much higher incidence (2.5\%) [9]. Unlike the patients from the previous studies, this study included patients who complained of chest or epigastric symptoms suggestive of EoE, including heartburn, dysphagia, epigastric pain, chest pain, acid regurgitation, food impaction, and vomiting [9]. Epidemiological studies on EoE performed in Europe and North America indicate that the incidence is on the rise. Two regional population-based studies in Switzerland revealed that the incidence and cumulative prevalence of EoE has increased rapidly over the past $10-20$ years, with a current prevalence of $24-43$ per 100,000 inhabitants $(0.024-0.043 \%)[10,11]$. Similarly, a Spanish study estimated a prevalence of 1 in 2,250 (0.04\%) [12]. The findings from studies using national databases in Europe and the USA suggest that the rising incidence has not yet reached a plateau [13-15]. There also appears to be a seasonal trend in the volume of disease diagnoses, and in many patients, EoE is a chronic relapsing disease [14].

Although EoE is increasingly prevalent, there are very little data on the prevalence and incidence of the other EGIDs. In the USA, the prevalence of EGE is estimated at
$22-28$ per 100,000 persons [16]. A US study estimated the prevalence ofEGE andEoCat 8.4/100,000 and3.3/100,000, respectively, with a predominance in females for these non-EoE conditions [17]. Given the strong immunological component in the disease pathology, it is not surprising that concomitant atopic diseases were observed in many patients, particularly in children [17]. There are very little epidemiological data on the prevalence and incidence of EGE in Asian countries [18], but there are likely to be differences in gastrointestinal symptoms between Asians and Caucasians [4]. These may be due to differences in the prevalence of infectious agents (such as Helicobacter pylori) as well as differences in dietary habits [4].

\section{Patient Characteristics}

The majority of EoE patients are men in their 20s or 30 s, although data from other studies have indicated later presentations [18-21]. A retrospective Australian study, which confirmed a diagnosis of EoE in 31 adults (24 men and 7 women), indicated that the mean age at diagnosis was 34 years [19], in contrast to a mean age at diagnosis of 49 years in a Japanese study [18]. In the Australian patients, symptoms (predominantly dysphagia) had been present for an average of 4.5 years prior to diagnosis [19]. The male predominance may be related to variations in a gene located on the $\mathrm{X}$-chromosome which have been associated with EoE. It is not surprising that among children, EoE is also more frequently observed in boys [22, 23].

EoE is strongly associated with certain allergic conditions, including food allergies, environmental allergies, asthma, and atopic dermatitis. An estimated $26-86 \%$ of adults and $42-93 \%$ of children diagnosed with EoE also have another allergic condition [14, 22, 24-31]. Related allergic disorders include asthma, allergic rhinitis, urticaria, hay fever, atopic dermatitis, food allergy, or drug allergies [29, 31]. A study in children with EoE indicated that $68 \%$ had a positive skin or radioallergosorbent test against a median of 7 food allergens [28]. A recent metaanalysis concluded that there is no clear link between EoE and celiac disease [32]. EoE was found with a high prevalence in patients with inherited connective tissue disorders [33]. Antibiotic use in infancy was associated with higher odds of having EoE [34]. In addition, cesarean delivery, preterm birth, and formula-only or mixed (infant formula and breast milk) feeding also showed trends toward increased odds for developing EoE [34].
Ishihara/Kinoshita/Schoepfer 
As its name implies, EGE can affect the entire gastrointestinal tract, from the stomach to the colon [35-38]. The clinical features of EGE are related to the location, extent, and layer(s) of bowel with eosinophilic infiltration [39]. Although studies have suggested that EGE has a predilection for the distal antrum and proximal small bowel, this may reflect sampling bias because of the greater accessibility of these areas for biopsy [39]. EGE can affect patients of any age, but it most frequently presents in the third through the fifth decade with a peak age of onset in the third decade [38]. Similar to EoE, a large proportion of patients with EGE (around 50\%) have a history of allergic diseases including asthma, defined food sensitivities, eczema, or rhinitis [38, 40,41]. Interestingly, a recent Japanese study revealed that the prevalence of infection by $H$. pylori was significantly lower in patients with EGE and EoE compared to control subjects, implying a role of Th1/ Th2 imbalance in the etiology of these disorders [42].

\section{Genetics}

Under normal conditions, the esophagus is devoid of eosinophils. Interestingly, the normal distribution of eosinophils in the gastrointestinal tract has only recently been thoroughly evaluated, including the definition of normal values in the different gastrointestinal segments. An increasing number of eosinophils from the stomach to the right-sided colon are observed with a decreasing number of eosinophils in the left-sided colon [43]. Eosinophils synthesize and secrete numerous important inflammatory and regulatory molecules including cytokines, chemokines, and growth factors that function in the regulation of the intestinal microenvironment [43]. Although the exact mechanisms through which eosinophils are involved in the pathogenesis of the EGIDs remain unknown, recent evidence suggests the dysregulation of specific cytokines in EoE and EGE [44]. Elevated plasma concentrations of IL-5 and IL-15 were observed in Japanese EoE and EGE patients compared to normal controls, a finding which correlated with blood eosinophil count [44]. A recent transcriptome analysis comparing the EoE gene signatures between Japanese and US patients identified several common IL-13-inducible and eosinophil-related genes, including eotaxin-3/CCL26, suggesting that the molecular pathogenic mechanisms of EoE may be similar in Asian and Western countries [45]. Genome-wide association studies have identified several loci associated with EoE, namely TSLP/WDR36, c11orf30, and STAT6 (also associated with atopic and autoimmune diseases), as well as
Table 1. Diagnostic criteria of EoE [48]

Presence of symptoms arising from esophageal dysfunction Biopsy findings $\geq 15$ eosinophils/high-power field under 8-week treatment with proton pump inhibitors (standard single or double dose)

Other causes of esophageal eosinophilia have been excluded (see table 2)

ANKRD27 and CAPN14 [46, 47]. The latter two loci are thought to be specific to EoE [47]. The current hypothesis for EoE suggests an interplay between allergic sensitization alongside an IL-13-inducible esophageal response that may involve CAPN14 [46]. Further studies are urgently needed in order to understand the molecular mechanisms involved and to identify new therapeutic targets.

\section{Diagnosis}

International guidelines for the diagnosis and management of EoE exist, but not yet for EGE and EoC. In Japan, a research group of the Ministry of Health, Labor and Welfare has recently announced recommendations for the diagnosis and management of EoE and EGE. In Switzerland, clinicians rely on the guidelines established by the American College of Gastroenterology (ACG) [48] and a recent consensus statement [49] for EoE. The diagnostic principles for EoE are based on the concept that this appears to be a chronic, immune-mediated disease defined clinically by symptoms related to esophageal dysfunction and histologically by eosinophil-driven inflammation [49]. The diagnostic criteria for EoE are summarized in table 1.

A diagnosis of EoE is based on findings of esophageal dysfunction, a dense eosinophilic infiltrate ( $\geq 15$ eosinophils/high-power field) that persists under an at least 8 -week treatment with proton pump inhibitors (using a standard or double dose), and exclusion of differential diagnoses that can also be associated with esophageal eosinophilia (table 2) [48]. In Japan, besides the clinical and histological pillars, endoscopic findings are frequently used to support an EoE diagnosis. Longitudinal furrows are the most frequently reported abnormality, observed in $52 \%$ of the patients, followed by white plaques and concentric rings [5-9, 18, 21, 50]. A recent Japanese study reported that longitudinal furrows corresponded to the highest positive and negative predictive values [9]. In Swit- 
Table 2. Differential diagnoses: other diseases that should be differentiated from EoE [52]

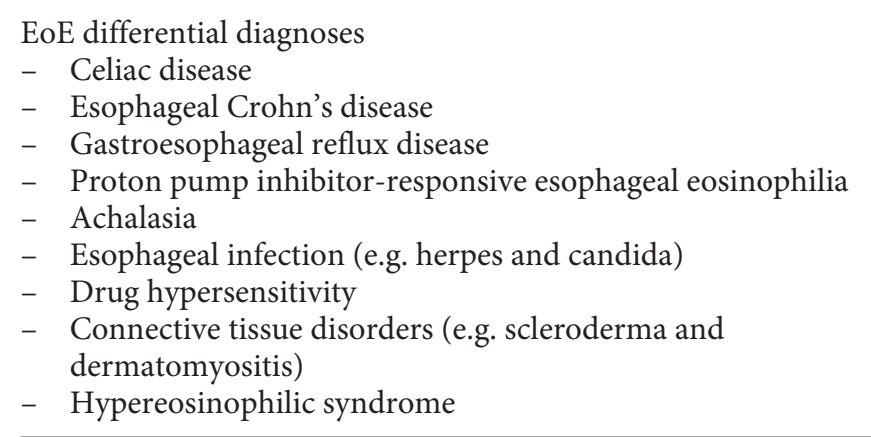

zerland, imaging (scans and endoscopic ultrasound) or functional exams ( $\mathrm{pH}$-metric studies and manometry) are not routinely used for diagnosis as these are unspecific. Of note, the ACG guidelines do not recommend systematic imaging by a thoracic scan or by endoscopic ultrasound for establishing an EoE diagnosis [48]. In both Asia and Europe, blood analyses are routinely performed. A workup by an allergist is recommended for every EoE patient (specific IgE, atopy patch testing, and skin prick testing).

Worldwide, similar criteria are used in the diagnosis of EGE and EoC. Peripheral blood eosinophilia and findings obtained from computed tomography or ultrasound are frequently used, since these tests are noninvasive and easily performed in clinical settings [18]. The presence of eosinophilic infiltration of the gastrointestinal tract upon biopsy and/or the presence of eosinophilic ascitic fluid, lack of involvement of other organs, and the absence of other known causes of intestinal eosinophilia are important criteria for establishing a diagnosis (table 3). An overview of the differential diagnoses for EGE is given in table 4. Endoscopic findings of mucosal disease are nonspecific and often include nodular or polypoid gastric mucosa, erythema, or erosions. Because the stomach and duodenum are the most commonly affected sites, endoscopic evaluation is typically limited to the upper gastrointestinal tract, although colonoscopy is recommended for patients with significant diarrhea. A key diagnostic criterion for mucosal EGE is the presence of a higher number of eosinophils than expected upon microscopic examination of gastrointestinal biopsies [51-53]. As there are no formally defined histological cutoff values for the number of eosinophils per high-power field for EGE, the diagnosis should be confirmed by an experienced pathologist to assess if the number of eosinophils is higher than expected for a particular area.
Table 3. Diagnostic criteria of EGE

Medical history and physical examination

- Presence of symptoms (abdominal pain, diarrhea, vomiting, dysphagia, and food impaction)

- Examination of the skin, eyes, nose, lymph nodes, and abdomen, in addition to performing a cardiovascular, respiratory, and neurologic examination

- Presence of ascites (eosinophilia in ascites)

- Gastrointestinal wall thickness (diagnosed by computed tomography, ultrasonography, or endoscopic ultrasound)

- Exclusion of differential diagnoses (see table 4)

Laboratory tests

- Peripheral blood eosinophilia

- Elevated serum IgE concentration

- Stool studies for parasites

- Ascitic fluid analyses (in patients with ascites)

- Renal function (serum chemistries, creatinine, and urinalysis)

- Hepatic function (liver enzymes and total bilirubin)

- Cardiac function (troponin and electrocardiogram); those with elevated troponin should have an echocardiogram

- Pulmonary function

Endoscopy and biopsies [42, 55-57]

- Common endoscopic findings: edema, erosion, and reddening

- Biopsies should be taken from both normal and abnormal appearing mucosa, as normal appearing mucosa can demonstrate eosinophilic inflammation

- Lamina propria eosinophilic infiltration: >20 eosinophils/ high-power field ${ }^{1}$

- Multiple biopsy samples (at least 4-5 biopsies per site) should be taken from both the stomach and small intestine with additional biopsies of areas with visual abnormalities

- In patients with mucosal disease, upper endoscopy with biopsy of the stomach and small intestine is diagnostic in at least $80 \%$ of patients; however, it is important to note that mucosal biopsies are normal in patients with muscular or subserosal disease

- Full-thickness biopsy: as negative endoscopic mucosal biopsies do not definitively rule out muscular or subserosal EGE, laparoscopic full-thickness biopsy is necessary to establish the diagnosis; in patients with bowel wall thickening and/or obstruction, this also serves to exclude an underlying malignancy

${ }^{1}$ Guideline by the Japanese Ministry of Health, Labor and Welfare. No other eosinophil thresholds are available.

\section{Treatment and Management}

A combination of dietary, pharmacological, and endoscopic interventions (esophageal dilation) are used for the treatment of EoE [54]. The use of pharmacological agents and dietary changes target the inflammation associated with EoE, whereas esophageal dilation addresses 
Table 4. Differential diagnoses: other diseases that should be differentiated from EGE

EGE differential diagnoses

- Intestinal parasites: infection with Ancylostoma, Anisakis, Ascaris, Strongyloides, Toxocara, Trichiura, Capillaria, Basidiobolomycosis, and Trichinella can cause gastrointestinal symptoms and peripheral eosinophilia; infection with the dog hookworm, Ancylostoma caninum, can mimic EGE clinically and pathologically with eosinophilic infiltration of the gut wall and ascites

- Malignancy: lymphoma, gastric cancer, and colon cancer

- Inflammatory bowel disease: Crohn's disease associated with peripheral eosinophilia and/or an eosinophil-rich tissue infiltrate (rare)

- Hypereosinophilic syndrome

- Polyarteritis nodosa

- Eosinophilic granulomatosis with polyangiitis

- Eosinophilic granuloma (Langerhans cell histiocytosis)

tissue remodeling and fibrotic complications in the esophagus. In both Asian and Western countries, the use of corticosteroids (topical and less frequently systemic) forms the cornerstone of treatment for EoE. The topical glucocorticoids budesonide and fluticasone are currently the first-line therapies of choice. In a cohort of adolescent and adult Swiss patients, treatment with oral budesonide was able to achieve a high degree of clinical and histological remission (80 and 90\%, respectively) [55]. An adverse effect that emerged from this study was an increased risk of oroesophageal candidiasis [55]. Data from the Swiss EoE cohort indicated that swallowed topical corticosteroid therapy with either budesonide or fluticasone reduced the risk of food bolus impactions [56]. Although systemic corticosteroids (i.e. prednisone or methylprednisolone) have been shown to rapidly resolve the symptoms of EoE, several problems have emerged in connection with their use. First, symptoms reappear after tapering off the steroid dose [57]. Second, there is concern over the adverse effects of long-term systemic corticosteroid use. Therefore, systemic treatments are reserved for patients with severe disease or who are refractory to topical agents. Through their anti-inflammatory effects, the use of proton pump inhibitors has been shown to ameliorate eosinophilia in the esophagus, yielding clinical and histological remission in up to $50 \%$ of EoE patients $[58,59]$. In this case, the term 'proton-pump inhibitor-responsive esophageal eosinophilia' should be used.

Since food allergies are commonly reported in patients with EoE, an important therapeutic approach involves the identification and elimination of dietary antigens
[57]. Milk products and wheat are the most commonly identified drivers of eosinophilic inflammation in the esophagus. Dietary therapy in EoE involves the use of elemental diets (amino acid-based formulas) and elimination diets. Elemental diets appear to be effective at achieving disease remission, but the poor taste and the need to forego all foods are a major barrier to long-term adherence. Elimination diets based on the avoidance of specific food allergens are effective in some patients, but the success of this strategy hinges on the predictive value of allergy testing in identifying the offending antigens [60]. In Switzerland, milk products and wheat products represent staple foods; thus, few patients are willing to adopt elimination diets but prefer long-term treatment with swallowed topical corticosteroids. Another treatment that is used in Switzerland is esophageal dilation. This is a safe and effective strategy that can provide long-lasting relief from EoE symptoms [61]. It should be noted that dilation has no effect on the underlying inflammation, and despite the high degree of patient acceptance, some experience postprocedural pain [61]. Esophageal dilation is associated with a low risk for esophageal perforation. Finally, patients may also participate in ongoing clinical trials, including those evaluating targeted therapies such as monoclonal antibodies against IL-13, IL-5, or IL-15.

For EGE, the treatment modalities are similar in Japan and Switzerland. Due to the rarity of this disease, there is a lack of randomized controlled trials to guide the choice of therapy. The use of local (i.e. budesonide) or systemic (i.e. prednisone) corticosteroids forms the mainstay of treatment. Unlike in EoE, the use of topical corticosteroids may not achieve symptom relief in the majority of EGE patients, since the affected areas lie deeper within the gastrointestinal tract. One of the problems is that the dosage and formulation of topical corticosteroids have yet to be defined for the use in EGE [62]. A case in point is the controlled ileal-release capsule formulation of budesonide, which delivers the drug to the ileum but is less effective for drug delivery to the stomach or duodenum [62]. The use of systemic corticosteroids is associated with adverse effects such as weight gain, steroid-induced diabetes, arterial hypertension, acne, and effects on the adrenal axis. Immunomodulatory agents (i.e. azathioprine) are often used for patients with steroid-refractory disease or for the management of recurrent disease flares. Azathioprine, however, is not without unwanted effects (i.e. drug-induced hepatitis, pancreatitis, and leukopenia). As for EoE, elimination diets have also been used with some success. The treatment modalities for EGE reflect a compromise between drug-related adverse effects and relief of disease symptoms. 


\section{Future Directions}

Due to the rarity of the EGIDs, many unanswered questions remain with respect to disease pathogenesis, classification, diagnosis, and treatment. Since 2009, the International Eosinophilic Esophagitis Activity Index (EEsAI) study group has been developing and validating instruments to assess the activity of EoE at the level of symptoms, quality of life, endoscopy, and histology. The development and validation of tools which can integrate patient-reported outcomes, quality of life, and endoscopic and histological activity in adult and pediatric EoE patients will be useful for clinical management. Since November 2015, EoE patients from several cantons of Switzerland have been systematically included into the prospective Swiss EoE Cohort Study (SEECS). The cre- ation of patient registries and the inclusion of patients in ongoing clinical trials will provide much-needed information on disease incidence, prevalence, natural history, and real-life efficacy of new treatments.

\section{Disclosure Statement}

A.S. received consulting fees and/or speaker fees and/or research grants from AstraZeneca AG (Switzerland), Aptalis Pharma Inc., Dr. Falk Pharma GmbH (Germany), GlaxoSmithKline AG, Nestlé S.A. (Switzerland), and Receptos Inc. Y.K. and S.I. received research grants from Daiichi-Sankyo Pharm, Takeda Pharm, Astellas Pharm, AstraZeneca KK, Mitsubishi Tanabe Pharm, and Eisai Pharm. Y.K. received honoraria or lecture fees from Daiichi-Sankyo Pharm, Takeda Pharm, Astellas Pharm, AstraZeneca KK, Zeria Pharm, Abbot, and Eisai Pharm.

\section{References}

1 Merves J, Muir A, Modayur CP, et al: Eosinophilic esophagitis. Ann Allergy Asthma Immunol 2014;112:397-403.

2 Ingle SB, Hinge Ingle CR: Eosinophilic gastroenteritis: an unusual type of gastroenteritis. World J Gastroenterol 2013;19:50615066.

-3 Alfadda AA, Storr MA, Shaffer EA: Eosinophilic colitis: epidemiology, clinical features, and current management. Therap Adv Gastroenterol 2011;4:301-309.

4 Ito J, Fujiwara T, Kojima R, Nomura I: Racial differences in eosinophilic gastrointestinal disorders among Caucasian and Asian. Allergol Int 2015;64:253-259.

5 Fujishiro H, Amano Y, Kushiyama Y, et al: Eosinophilic esophagitis investigated by upper gastrointestinal endoscopy in Japanese patients. J Gastroenterol 2011;46:1142-1144.

-6 Fujiwara Y, Sugawa T, Tanaka F, et al: A multicenter study on the prevalence of eosinophilic esophagitis and PPI-responsive esophageal eosinophilic infiltration. Intern Med 2012;51:3235-3239.

7 Hori K, Watari J, Fukui H, et al: Do endoscopic features suggesting eosinophilic esophagitis represent histological eosinophilia? Dig Endosc 2014;26:156-163.

8 Tomomatsu Y, Yoshino J, Inui K, et al: Clinical features of eosinophilic esophagitis: ten Japanese cases. Dig Endosc 2013;25:117-124.

-9 Shimura S, Ishimura N, Tanimura T, et al: Reliability of symptoms and endoscopic findings for diagnosis of esophageal eosinophilia in a Japanese population. Digestion 2014;90: 49-57.

10 Giriens B, Yan P, Safroneeva E, et al: Escalating incidence of eosinophilic esophagitis in Canton of Vaud, Switzerland, 1993-2013: a population-based study. Allergy 2015;70: 19 Croese J, Fairley SK, Masson JW, et al: Clinical $1633-1639$.

11 Hruz P, Straumann A, Bussmann C, et al: Escalating incidence of eosinophilic esophagitis: a 20-year prospective, population-based study in Olten County, Switzerland. J Allergy Clin Immunol 2011;128:1349-1350.

12 Arias A, Lucendo AJ: Prevalence of eosinophilic oesophagitis in adult patients in a central region of Spain. Eur J Gastroenterol Hepatol 2013;25:208-212.

13 Kapel RC, Miller JK, Torres C, et al: Eosinophilic esophagitis: a prevalent disease in the United States that affects all age groups. Gastroenterology 2008;134:1316-1321.

14 Prasad GA, Alexander JA, Schleck CD, et al: Epidemiology of eosinophilic esophagitis over three decades in Olmsted County, Minnesota. Clin Gastroenterol Hepatol 2009;7: 1055-1061.

15 van Rhijn BD, Verheij J, Smout AJ, Bredenoord AJ: Rapidly increasing incidence of eosinophilic esophagitis in a large cohort. Neurogastroenterol Motil 2013;25:47-52.

16 Spergel JM, Book WM, Mays E, et al: Variation in prevalence, diagnostic criteria, and initial management options for eosinophilic gastrointestinal diseases in the United States. J Pediatr Gastroenterol Nutr 2011;52:300-306.

17 Jensen ET, Martin CF, Kappelman MD, Dellon ES: Prevalence of eosinophilic gastritis, gastroenteritis, and colitis: estimates from a national administrative database. J Pediatr Gastroenterol Nutr 2016;62:36-42.

18 Kinoshita Y, Furuta K, Ishimaura N, et al: Clinical characteristics of Japanese patients with eosinophilic esophagitis and eosinophilic gastroenteritis. J Gastroenterol 2013;48: 333-339. and endoscopic features of eosinophilic esophagitis in adults. Gastrointest Endosc 2003;58:516-522.

20 Dellon ES, Jensen ET, Martin CF, et al: Prevalence of eosinophilic esophagitis in the United States. Clin Gastroenterol Hepatol 2014;12: 589-596.

21 Kinoshita Y, Ishimura N, Oshima N, Ishihara S: Systematic review: eosinophilic esophagitis in Asian countries. World J Gastroenterol 2015;21:8433-8440.

22 Noel RJ, Putnam PE, Rothenberg ME: Eosinophilic esophagitis. N Engl J Med 2004;351: 940-941.

23 Franciosi JP, Tam V, Liacouras CA, Spergel JM: A case-control study of sociodemographic and geographic characteristics of 335 children with eosinophilic esophagitis. Clin Gastroenterol Hepatol 2009; 7:415419.

24 Almansa C, Krishna M, Buchner AM, et al: Seasonal distribution in newly diagnosed cases of eosinophilic esophagitis in adults. Am J Gastroenterol 2009;104:828-833.

25 Dohil R, Newbury R, Fox L, et al: Oral viscous budesonide is effective in children with eosinophilic esophagitis in a randomized, placebocontrolled trial. Gastroenterology 2010;139: 418-429.

26 Erwin EA, James HR, Gutekunst HM, et al: Serum IgE measurement and detection of food allergy in pediatric patients with eosinophilic esophagitis. Ann Allergy Asthma Im-

27 Moawad FJ, Veerappan GR, Lake JM, et al: Correlation between eosinophilic oesophagitis and aeroallergens. Aliment Pharmacol Ther 2010;31:509-515. munol 2010;104:496-502. 
28 Orenstein SR, Shalaby TM, Di LC, et al: The spectrum of pediatric eosinophilic esophagitis beyond infancy: a clinical series of 30 children. Am J Gastroenterol 2000;95:1422-1430.

-29 Roy-Ghanta S, Larosa DF, Katzka DA: Atopic characteristics of adult patients with eosinophilic esophagitis. Clin Gastroenterol Hepatol 2008;6:531-535.

-30 Spergel JM, Brown-Whitehorn TF, Beausoleil $\mathrm{JL}$, et al: 14 years of eosinophilic esophagitis: clinical features and prognosis. J Pediatr Gastroenterol Nutr 2009;48:30-36.

-31 Vitellas KM, Bennett WF, Bova JG, et al: Idiopathic eosinophilic esophagitis. Radiology 1993; 186:789-793.

-32 Lucendo AJ, Arias A, Tenias JM. Systematic review: the association between eosinophilic oesophagitis and coeliac disease. Aliment Pharmacol Ther 2014;40:422-434.

-33 Abonia JP, Wen T, Stucke EM, et al: High prevalence of eosinophilic esophagitis in patients with inherited connective tissue disorders. J Allergy Clin Immunol 2013;132:378386.

34 Jensen ET, Kappelman MD, Kim HP, et al: Early life exposures as risk factors for pediatric eosinophilic esophagitis. J Pediatr Gastroenterol Nutr 2013;57:67-71.

-35 Haberkern CM, Christie DL, Haas JE: Eosinophilic gastroenteritis presenting as ileocolitis. Gastroenterology 1978;74:896-899.

36 Matsushita M, Hajiro K, Morita Y, et al: Eosinophilic gastroenteritis involving the entire digestive tract. Am J Gastroenterol 1995;90: 1868-1870.

- 37 Schoonbroodt D, Horsmans Y, Laka A, et al Eosinophilic gastroenteritis presenting with colitis and cholangitis. Dig Dis Sci 1995;40: 308-314.

38 Talley NJ, Shorter RG, Phillips SF, Zinsmeister AR: Eosinophilic gastroenteritis: a clinicopathological study of patients with disease of the mucosa, muscle layer, and subserosal tissues. Gut 1990;31:54-58.

39 Klein NC, Hargrove RL, Sleisenger MH, Jeffries GH: Eosinophilic gastroenteritis. Medicine (Baltimore) 1970;49:299-319.
40 Caldwell JH, Tennenbaum JI, Bronstein HA: Serum IgE in eosinophilic gastroenteritis. Response to intestinal challenge in two cases. $\mathrm{N}$ Engl J Med 1975;292:1388-1390.

41 Yun MY, Cho YU, Park IS, et al: Eosinophilic gastroenteritis presenting as small bowel obstruction: a case report and review of the literature. World J Gastroenterol 2007;13:17581760.

42 Furuta K, Adachi K, Aimi M, et al: Case-control study of association of eosinophilic gastrointestinal disorders with Helicobacter py lori infection in Japan. J Clin Biochem Nutr 2013;53:60-62.

43 Matsushita T, Maruyama R, Ishikawa N, Harada Y, Araki A, Chen D, Tauchi-Nishi P, Yuki T, Kinoshita Y: The number and distribution of eosinophils in the adult human gastrointestinal tract: a study and comparison of racial and environmental factors. Am J Surg Pathol 2015;39:521-527.

44 Kinoshita Y, Furuta K, Ishimura N, Ishihara $\mathrm{S}$ : Elevated plasma cytokines in Japanese patients with eosinophilic esophagitis and gastroenteritis. Digestion 2012;86:238-243.

45 Shoda T, Morita H, Nomura I, et al: Comparison of gene expression profiles in eosinophilic esophagitis (EoE) between Japan and Western countries. Allergol Int 2015;64:260-265.

46 Kottyan LC, Davis BP, Sherrill JD, et al: Genome-wide association analysis of eosinophilic esophagitis provides insight into the tissue specificity of this allergic disease. Nat Genet 2014;46:895-900.

47 Sleiman PM, Wang ML, Cianferoni A, et al: GWAS identifies four novel eosinophilic esophagitis loci. Nat Commun 2014;5:5593.

48 Dellon ES, Gonsalves N, Hirano I, et al: ACG clinical guideline: evidenced based approach to the diagnosis and management of esophageal eosinophilia and eosinophilic esophagitis (EoE). Am J Gastroenterol 2013;108:679-692.

49 Liacouras CA, Furuta GT, Hirano I, et al: Eosinophilic esophagitis: updated consensus recommendations for children and adults. J Allergy Clin Immunol 2011;128:3-20.

50 Ishimura N, Shimura S, Jiao D, et al: Clinical features of eosinophilic esophagitis: differences between Asian and Western populations. J Gastroenterol Hepatol 2015;30(suppl 1):71-77.
Katz AJ, Goldman H, Grand RJ: Gastric mucosal biopsy in eosinophilic (allergic) gastroenteritis. Gastroenterology 1977;73:705-709.

52 Lee CM, Changchien CS, Chen PC, et al: Eosinophilic gastroenteritis: 10 years experience. Am J Gastroenterol 1993;88:70-74.

53 Lee M, Hodges WG, Huggins TL, Lee EL: Eosinophilic gastroenteritis. South Med J 1996; 89:189-194.

54 Park H: An overview of eosinophilic esophagitis. Gut Liver 2014;8:590-597.

- 55 Straumann A, Conus S, Degen L, et al Budesonide is effective in adolescent and adult patients with active eosinophilic esophagitis. Gastroenterology 2010;139:15261537.

56 Kuchen T, Straumann A, Safroneeva E, et al: Swallowed topical corticosteroids reduce the risk for long-lasting bolus impactions in eosinophilic esophagitis. Allergy 2014;69:12481254.

57 Dellon ES, Liacouras CA: Advances in clinical management of eosinophilic esophagitis. Gastroenterology 2014;147:1238-1254.

58 Munday W, Zhang X: Proton pump inhibitor responsive esophageal eosinophilia, a distinct disease entity? World J Gastroenterol 2014; 20:10419-10424.

59 Lucendo AJ, Arias A, Molina-Infante J: Efficacy of proton pump inhibitor drugs for inducing clinical and histologic remission in patients with symptomatic esophageal eosinophilia: a systematic review and meta-analysis. Clin Gastroenterol Hepatol 2016;14:13-22.

-60 Lucendo AJ: Meta-analysis-based guidance for dietary management in eosinophilic esophagitis. Curr Gastroenterol Rep 2015;17: 464

61 Schoepfer AM, Gonsalves N, Bussmann C, et al: Esophageal dilation in eosinophilic esophagitis: effectiveness, safety, and impact on the underlying inflammation. Am J Gastroenterol 2010;105:1062-1070.

62 Prussin C: Eosinophilic gastroenteritis and related eosinophilic disorders. Gastroenterol Clin North Am 2014;43:317-327.
Eosinophilic Esophagitis, Gastroenteritis, and Colitis in the East and West
Inflamm Intest Dis 2016;1:63-69 